\title{
Effect of quasiresonant dynamics on the predissociation of van der Waals molecules
}

\section{Citation}

Forrey, R. C., N. Balakrishnan, A. Dalgarno, M. R. Haggerty, and E. J. Heller. 2001. "Effect of Quasiresonant Dynamics on the Predissociation of van Der Waals Molecules." Physical Review A 64 (2). https://doi.org/10.1103/physreva.64.022706.

\section{Permanent link}

http://nrs.harvard.edu/urn-3:HUL.InstRepos:41417410

\section{Terms of Use}

This article was downloaded from Harvard University's DASH repository, and is made available under the terms and conditions applicable to Other Posted Material, as set forth at http:// nrs.harvard.edu/urn-3:HUL.InstRepos:dash.current.terms-of-use\#LAA

\section{Share Your Story}

The Harvard community has made this article openly available.

Please share how this access benefits you. Submit a story.

Accessibility 


\title{
Effect of quasiresonant dynamics on the predissociation of van der Waals molecules
}

\author{
R. C. Forrey, ${ }^{1,2}$ N. Balakrishnan, ${ }^{2}$ A. Dalgarno, ${ }^{2}$ M. R. Haggerty, ${ }^{3}$ and E. J. Heller ${ }^{3,4}$ \\ ${ }^{1}$ Penn State University, Berks-Lehigh Valley College, Reading, Pennsylvania 19610-6009 \\ ${ }^{2}$ Harvard-Smithsonian Center for Astrophysics, 60 Garden Street, Cambridge, Massachusetts 02138 \\ ${ }^{3}$ Department of Physics, Harvard University, Cambridge, Massachusetts 02138 \\ ${ }^{4}$ Department of Chemistry, Harvard University, Cambridge, Massachusetts 02138
}

(Received 16 November 2000; published 5 July 2001)

\begin{abstract}
Rotational and vibrational distributions of zero-temperature collisional rate coefficients for atom-diatom scattering are used together with effective range theory to obtain lifetimes for predissociation. High-order indirect potential coupling in the quantum-mechanical calculation is interpreted using a simple classical picture that describes the quasiresonant dynamics of atom-diatom collisions by the conservation of classical action. The importance of closed channel thresholds in determining the structure of the distributions and the balance between momentum gap and near-resonant effects is discussed.
\end{abstract}

DOI: 10.1103/PhysRevA.64.022706

PACS number(s): $34.10 .+\mathrm{x}$

\section{INTRODUCTION}

The possibility to cool and trap molecules [1-11] provides a unique opportunity to study collisions between atoms and molecules at very low translational energies [12-16] and may allow experimental detection of very narrow predissociation decay widths [13]. Vibrational predissociation will play an important role in the relaxation of vibrationally excited trapped molecules when the density of surrounding atoms is high and may be useful in determining Feshbach resonance parameters for ultracold atom-molecule collisions [13]. Calculations have demonstrated that very efficient and specific rovibrational transitions occur in the limit of zero temperature [14]. The dynamics that produce these so-called quasiresonant transitions can be expected to influence the predissociation of van der Waals molecules.

The importance of near-resonant effects in vibrational and rotational predissociation of van der Waals molecules has long been recognized [17-22]. For a van der Waals molecule consisting of an atom and a diatom, the internal energy of the diatom is converted into translational energy of the fragments. In pure vibrational predissociation where all of the diatom's vibrational energy is converted into translational energy, the predissociation widths are very small due to the large number of oscillations in the final-state continuum wave function. This so-called "momentum gap" effect is reduced when some of the diatom's vibrational energy is converted to rotational energy. A near-resonant process of this kind generally requires a large change in the rotational quantum number of the diatom. If the van der Waals molecule has weak anisotropy, the direct bound-continuum potential coupling is very small. In this case, the higher-order indirect potential coupling is controlling the predissociation [21]. However, a detailed understanding of the mechanism underlying the high-order indirect potential coupling has not been given.

Typically, the balance between the momentum gap and near resonant effects will produce an oscillatory rotational distribution for the partial predissociation widths of van der Waals molecules that have little or no internal angular momentum. It has been suggested [21] that the oscillatory rota- tional distributions could be a rotational rainbow phenomenon. This description requires that the rotational period of the diatom be longer than the time required for the fragments to separate.

In the present work, we consider the opposite case where the rotational period is short compared to the time required for the fragments to separate. For the most weakly bound state of the van der Waals complex, we find that the process of predissociation is controlled by the same classical dynamical quasiresonant transfer of energy that is found in ultracold atom-diatom collisions [14]. To demonstrate this finding, we calculate zero-temperature quenching rate coefficients for $\mathrm{He}$ collisions with $\mathrm{H}_{2}$ using the general purpose scattering program MOLSCAT [23] and the potential energy surface of Muchnick and Russek [24]. Effective range theory is used to relate the rate coefficients to the predissociation lifetimes. Threshold behavior is explained using a perturbative description of the scattering matrix elements.

\section{THEORY}

The calculation of predissociation lifetimes of weakanisotropy van der Waals molecules using perturbation theory has been the subject of several detailed investigations $[20,21]$. The studies concluded that perturbation theory calculations could successfully model the qualitative features of numerically exact close-coupling results, but could not provide results that were quantitatively accurate $[20,21]$. The reason was due to the difficulty in achieving an adequate representation of the bound state wave function and the neglect of potential coupling between open channels. This can be understood by considering the simplest type of perturbative scheme which is often referred to as the space-fixed distortion (SFD) method. In the zeroth-order approximation, the wave functions are computed by neglecting the nondiagonal matrix elements $V_{i f}$, and the decay process is calculated using the standard rule

$$
\Gamma_{i}=\sum_{f} \Gamma_{i, f}=2 \pi \sum_{f} g(f)\left|\int \chi_{i} V_{i f} \chi_{f} d \tau\right|^{2}
$$


where $\chi_{i}$ and $\chi_{f}$ are the unperturbed radial functions and $g(f)$ is the degree of degeneracy of the final state. If the momentum transfer is large, then the final continuum wave function will have many oscillations in the region of overlap, and the decay width will be small. In addition, the matrix element (1) will be very sensitive to small variations of the bound state wave function. Therefore a potential source of error in perturbation theory is an inadequate representation of the bound state wave function [21].

The other major source of error in perturbation theory comes from neglecting indirect potential coupling [20,21]. As stated in the introduction, the largest partial widths are typically found for final states for which the amount of transferred momentum is small. The direct bound-continuum potential coupling elements $V_{i f}$ that allow low-momentum transfer, however, arise from high-order terms in a Legendre expansion of the anisotropy of the intermolecular potential. These terms are very small for weak anisotropy van der Waals molecules suggesting that the predissociation width will be small unless there is a substantial contribution coming from the indirect intermediate potential couplings. In this case, the perturbative expression (1) provides a poor approximation to the predissociation width due to its neglect of the indirect coupling terms. It is possible to construct more sophisticated perturbation theories such as the secular equation perturbation theory for the open channels (SEPTOC) that are more accurate than the SFD method [20]. At this stage, however, the utility in using perturbation theory to gain a qualitative understanding of the physics of predissociation becomes limited.

A numerically exact procedure for computing the predissociation lifetimes is to solve a set of coupled channel equations for energies below threshold. The $S$ matrix is then diagonalized and the eigenphase sum is differentiated with respect to energy to obtain the resonance widths. This approach was taken [13] in order to establish the validity of multichannel effective range expansions for weakly bound complexes. The inverse of the predissociation lifetime of the most weakly bound state of the van der Waals complex was found to be well approximated by [13]

$$
\tau_{v j}^{-1}=\frac{1}{2 \pi r_{v j}\left|a_{v j}\right|^{2}}\left\{\left[1-\frac{2 \alpha_{v j} r_{v j}}{\left|a_{v j}\right|^{2}}\right]^{-1 / 2}-1\right\} \lim _{T \rightarrow 0} R_{v j}(T)
$$

where

$$
R_{v j}(T)=\sum_{v^{\prime} j^{\prime}} R_{v j \rightarrow v^{\prime} j^{\prime}}(T)=\frac{4 \pi \hbar \beta_{v j}}{\mu}
$$

is the total quenching rate coefficient, $\alpha_{v j}$ and $\beta_{v j}$ are the real and imaginary parts of the scattering length $a_{v j}=\alpha_{v j}$ $-i \beta_{v j}$, and $r_{v j}$ is the effective range for the diatomic level labeled by vibrational quantum number $v$ and rotational quantum number $j$. It is assumed in this analysis that the end-over-end angular momentum of the complex is zero and the vibrational stretching quantum number is the largest possible integer that allows the complex to be bound. The scat-

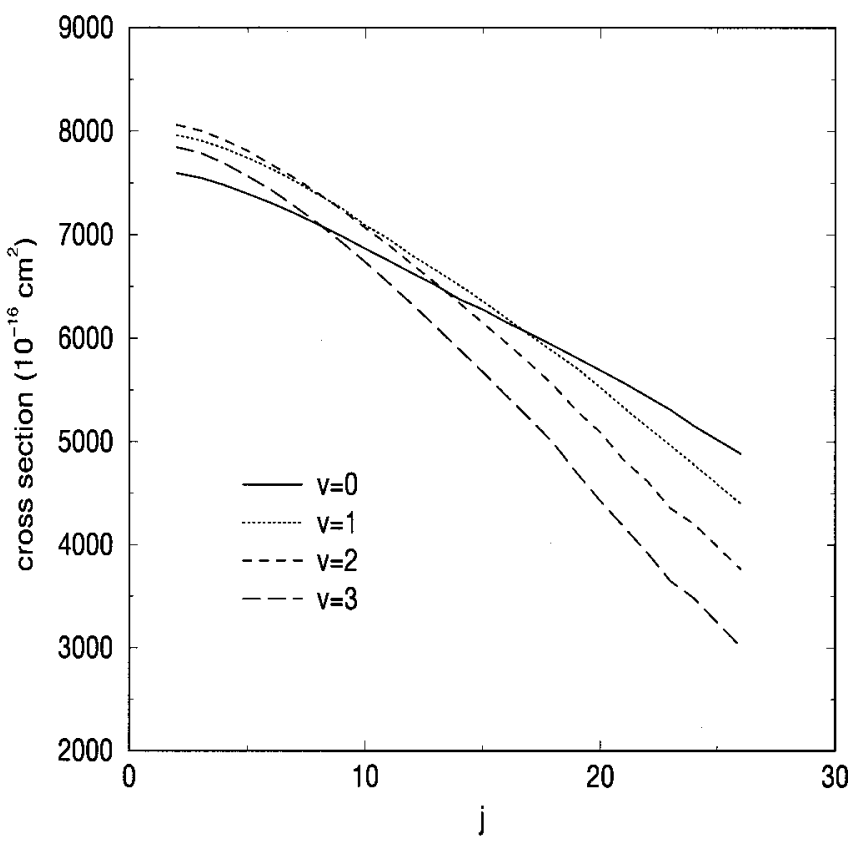

FIG. 1. Zero-energy elastic scattering cross sections for ${ }^{4} \mathrm{He}+\mathrm{H}_{2}(v, j)$ as a function of the initial vibrational and rotational quantum numbers $v$ and $j$.

tering length approximation may be obtained by setting the effective range parameter $\boldsymbol{r}_{v j}$ equal to zero in Eq. (2), yielding

$$
\tau_{v j}^{-1}=\frac{1}{2 \pi \alpha_{v j}^{3}} \lim _{T \rightarrow 0} R_{v j}(T)
$$

Typically, the real part of the scattering length is much larger than the imaginary part. This allows $\alpha_{v j}$ to be obtained directly from the zero-energy elastic scattering cross section

$$
\sigma_{v j \rightarrow v j}=4 \pi\left(\alpha_{v j}^{2}+\beta_{v j}^{2}\right) \approx 4 \pi \alpha_{v j}^{2} .
$$

Figure 1 shows zero-energy elastic scattering cross sections for ${ }^{4} \mathrm{He}+\mathrm{H}_{2}(v, j)$ as a function of the initial vibrational and rotational quantum numbers $v$ and $j$. The figure shows that $\alpha_{v j}$ decreases as $j$ is increased and the rate of decrease is greater as $v$ is increased. Generally, the variation of $\alpha_{v j}$ with $j$ is slow enough that the predissociation lifetimes are controlled by the total quenching rate coefficients $R_{v j}(T)$.

The multichannel effective range theory described above provides a link between the dynamics of quasiresonant (QR) scattering and the vibrational predissociation of weakly bound complexes. We have shown [14] that the quenching rate coefficients $R_{v j \rightarrow v^{\prime} j^{\prime}}(T)$ are strongly influenced by classical dynamics in the $T \rightarrow 0$ limit. The general rule followed by quasiresonant vibration-rotation (QRVR) transitions is

$$
\Delta I=n_{j} \Delta_{j}+n_{v} \Delta v=0,
$$

where $I=n_{j} j+n_{v} v$ is the conserved action and $n_{j}$ and $n_{v}$ are small integers. When the vibrational and rotational motion are in approximate low-order resonance, the condition 


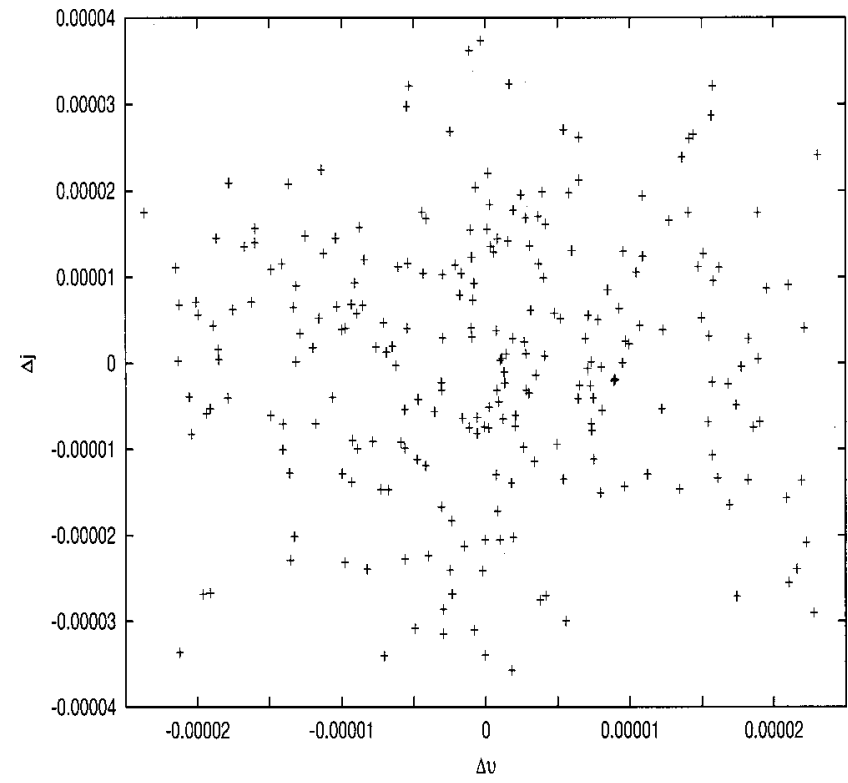

FIG. 2. A scatter plot of $\Delta j$ versus $\Delta v$ for $v_{i}=2, j_{i}=8$, and $E$ $=10^{-5}$ atomic units. The frequency ratio for this case is $\omega_{v} / \omega_{j}$ $\approx 4.5$ and there is no correlation between $\Delta j$ and $\Delta v$.

$$
n_{v} / n_{j} \approx \omega_{v} / \omega_{j}
$$

also holds, where $\omega_{v}$ and $\omega_{j}$ are the classical vibrational and rotational frequencies of the diatom. In this case, essentially all of the classical trajectories will obey the quasiresonant rule (6) for a single $\left(n_{v}, n_{j}\right)$ pair [25]. The classical behavior will persist as $T \rightarrow 0$ if $\Delta v$ and $\Delta j$ are allowed to take on noninteger values [14]. This is demonstrated in Figs. 2 and 3. Figure 2 shows a scatter plot of $\Delta v$ versus $\Delta j$ for an initial rotational level $j=8$ at a collision energy of $10^{-5}$ atomic

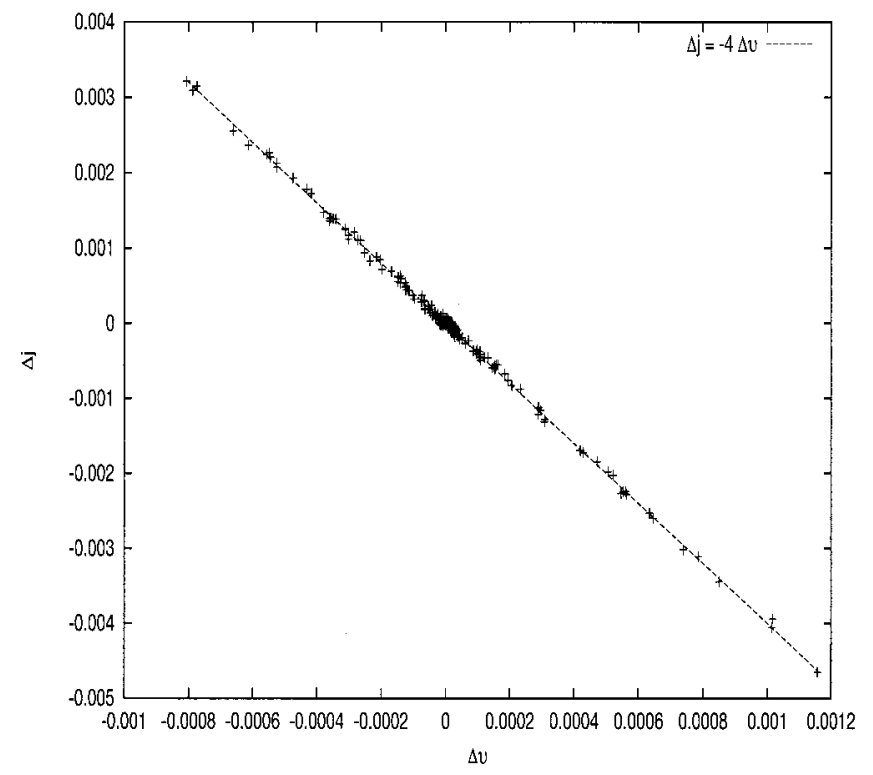

FIG. 3. A scatter plot of $\Delta j$ versus $\Delta v$ for $v_{i}=2, j_{i}=9$ and $E$ $=10^{-5}$ atomic units. The frequency ratio for this case is $\omega_{v} / \omega_{j}$ $\approx 4$ and there is strong correlation between $\Delta j$ and $\Delta v$. units. The ratio of vibrational frequency to rotational frequency is approximately equal to 4.5 for this case. Because this rotational level is unable to satisfy condition (7) there is no correlation between $\Delta v$ and $\Delta j$. When $j=9$, however, the ratio of vibrational frequency to rotational frequency is approximately equal to 4 and there is strong correlation between $\Delta v$ and $\Delta j$. This is shown in Fig. 3 along with the straight line corresponding to $\Delta j=-4 \Delta v$.

The correlation between $\Delta v$ and $\Delta j$ will persist in the quantum-mechanical calculations when there is enough energy that the quasiresonant channel is open. The conservation of action therefore provides a qualitative understanding of the mechanism underlying the high-order potential coupling that governs predissociation. The momentum gap argument is also explained by the classical analysis. Equations (6) and (7) yield

$$
\Delta E_{\mathrm{int}}=\frac{\partial H}{\partial v} \Delta v+\frac{\partial H}{\partial j} \Delta j=\hbar \omega_{v} \Delta v+\hbar \omega_{j} \Delta j \approx 0
$$

which is the condition that the internal energy of the diatom is approximately constant, or equivalently, that the momentum gap is as small as possible.

QRVR energy transfer in atom-diatom collisions at high energies has been described as a series of collisionettes [25]. Each collisionette resembles a separate collision that occurs when the rapidly rotating diatom is stretched to its outer turning point and is nearly collinear with the atom. Because the molecules are fully stretched, collisionettes can occur for large impact parameters and produce large cross sections [25]. In between each collisionette, however, the interaction potential decreases by several orders of magnitude. We have shown [14] that the collisionette picture needs to be modified in the $T \rightarrow 0$ limit. The distinct collisionettes are replaced by a strong modulation of the interaction potential at the characteristic frequency of the quasiresonant transition. Whereas the high-order potential coupling description is very complicated and difficult to understand, the modulation of the timedependent potential provides a simplified picture of the dynamics. The interaction potential alternates between positive and negative values and often binds the atom and diatom together for several successive subcollisions. Figure 4 illustrates such a long-time classical collision. The number of subcollisions depends on the choice of initial conditions. Since each subcollision follows the quasiresonant rule separately, the entire collision process does not depend on the initial conditions and therefore obeys the quasiresonant rule.

\section{RESULTS}

Figures 5 and 6 show zero-temperature quenching rate coefficients as a function of initial vibrational and rotational quantum numbers $v$ and $j$. It was shown previously [14] that rotational distributions like those shown in Figs. 5 and 6 had classical analogs that were a direct result of the correlation between $\Delta v$ and $\Delta j$ described above. A major difference, however, between the classical and quantum calculations arises at ultracold temperatures because it is not possible to 


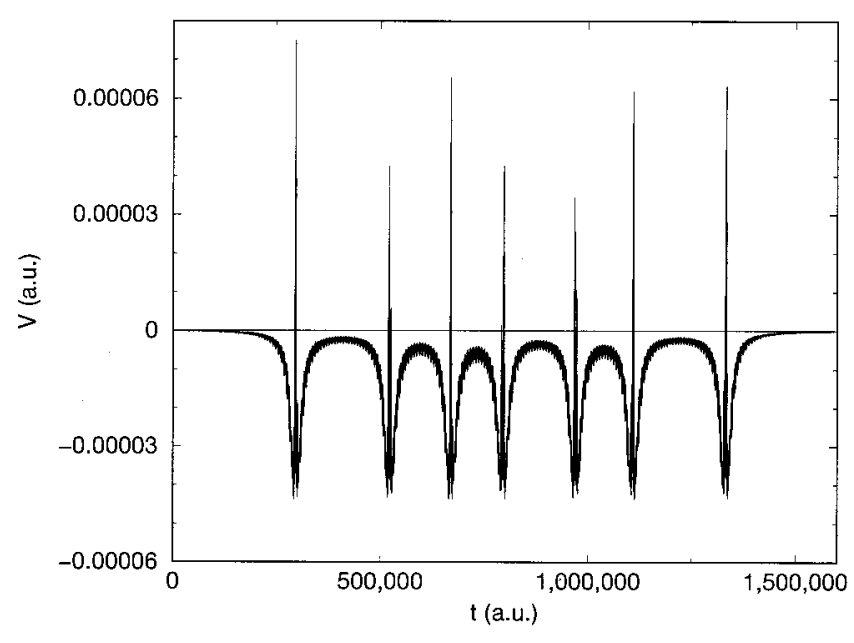

FIG. 4. A plot of the interaction potential versus time. The modulation occurs at the characteristic frequency of the quasiresonant transition. Seven subcollisions are contained within the total collision for this plot. Because each subcollision separately obeys the quasiresonant rule, the total collision preserves the correlation between $\Delta j$ and $\Delta v$.

have fractional changes in $v$ and $j$. Therefore many of the quasiresonant channels are closed. Furthermore, when quantum transitions are energetically barely allowed, they are subject to threshold behavior. The threshold structure in the rate coefficients will have a strong effect on the lifetimes. Using the results of Figs. 5 and 6, we computed predissociation lifetimes for several vibrational levels as a function of $j$. The results for $v=0$ and $v=2$ are shown in Fig. 7. For each of the calculations, the scattering length approximation given by Eq. (4) was used to compute the lifetimes. The effect of quasiresonant dynamics can be seen when $7<j<12$ and 20 $<j<25$. When $j=12$ there is a sharp decrease in both the $v=0$ and $v=2$ lifetimes. This is due to strong quasiresonant

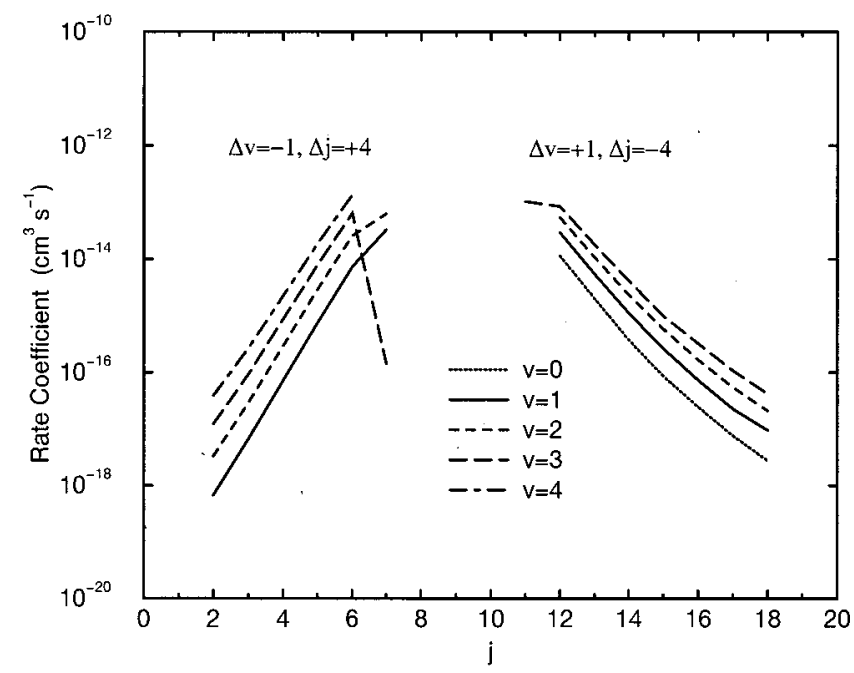

FIG. 5. Quantum-mechanical calculations of the zerotemperature rate coefficients for ${ }^{4} \mathrm{He}+\mathrm{H}_{2}(v, j)$ as a function of the initial vibrational and rotational quantum numbers $v$ and $j$.

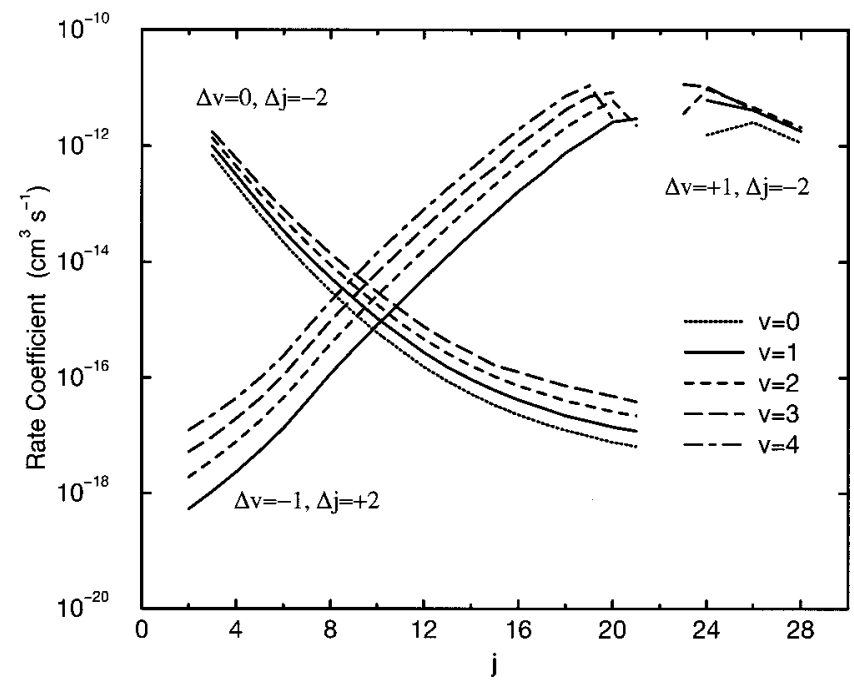

FIG. 6. Quantum-mechanical calculations of the zerotemperature rate coefficients for ${ }^{4} \mathrm{He}+\mathrm{H}_{2}(v, j)$ as a function of the initial vibrational and rotational quantum numbers $v$ and $j$.

$\Delta v=1, \Delta j=-4$ transitions (see Fig. 5). The $v=2$ curve shows a similar structure when $j=7$ which is due to the $\Delta v=-1, \Delta j=4$ quasiresonant transition. This symmetry is missing in the $v=0$ curve since $\Delta v=-1$ transitions are not allowed. The $v=2$ curve shows a second symmetric structure centered about $j=22$. This structure is due to the quasiresonant $\Delta v=-1, \Delta j=2$ and $\Delta v=1, \Delta j=-2$ transitions that are energetically allowed when $j=21$ and $j=23$ but are forbidden when $j=22$. The $v=0$ curve shows a sharp decrease when $j=24$ due to the opening of the $\Delta v=1, \Delta j$ $=-2$ quasiresonant channel. As before, the $v=0$ curve is asymmetric due to inaccessible $\Delta v=-1$ transitions. The predissociation lifetimes for other vibrational levels are very similar to the ones shown for $v=2$. Generally, the largest

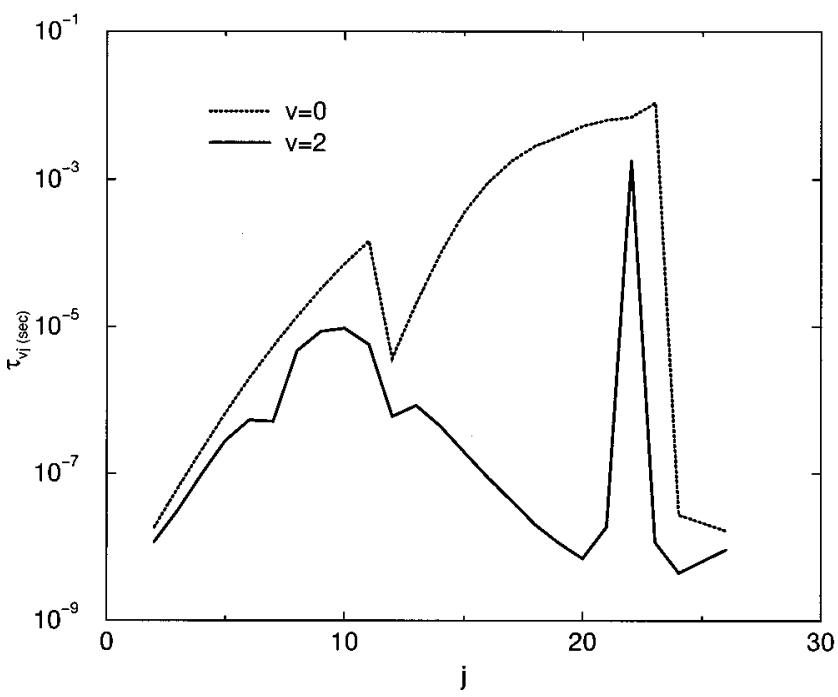

FIG. 7. Quantum-mechanical calculations of the predissociation lifetimes for ${ }^{4} \mathrm{He} \cdots \mathrm{H}_{2}(v, j)$ as a function of the initial rotational quantum number $j$. 
lifetimes occur for quasiresonant channels that are energetically closed.

In the vicinity of a threshold, the classical mechanics no longer applies and it is desirable to find a simple description that is fully quantum mechanical. Because perturbation theory failed for bound-free transitions, we expect the same to be true for free-free transitions. Nevertheless, the sudden decrease in the zero-temperature quenching rate coefficient at values of $j$ near thresholds (e.g., the sharp $v=3$ feature shown in Fig. 5) may be understood qualitatively by considering the Born approximation for the inelastic $K$-matrix element,

$$
K_{i f}=-\frac{2 \mu}{\hbar^{2}} \sqrt{k_{i} k_{f}} \int_{0}^{\infty} j_{l_{i}}\left(k_{i} r\right) V(r) j_{l_{f}}\left(k_{f} r\right) r^{2} d r
$$

where $k_{i}$ and $k_{f}$ are the respective wave numbers and $l_{i}$ and $l_{f}$ are the respective orbital angular momenta in the initial and final channels. For a short-range potential, the spherical Bessel functions in the integrand of Eq. (9) may be expanded in a power series. The $K$-matrix element then behaves as $O\left(k_{i}^{l_{i}+1 / 2} k_{f}^{l_{f}+1 / 2}\right)$ with $l_{i}=0$ for ultracold collisions. The zero-temperature rate coefficients are independent of $k_{i}$ and the discussion of threshold behavior is given in terms of $k_{f}$. For $l_{f}=2$ and $l_{f}=4$, the respective $K$-matrix elements vanish as $k_{f}^{5 / 2}$ and $k_{f}^{9 / 2}$ for small $k_{f_{5}}$. The inelastic scattering cross sections therefore vanish as $k_{f}^{5}$ and $k_{f}^{9}$ for small $k_{f}$, and we see sharp decreases in the zero-temperature rate coefficients near the thresholds for forbidden transitions.

The exact $k_{f}$ dependence of the cross sections may be modified for systems that possess a significant long-range potential. For a van der Waals potential of the form $V(r)$ $=C / r^{6}$, the integral (9) may be performed analytically. The result is

$$
\begin{aligned}
K_{i f}= & -\frac{\mu C}{\hbar^{2}} \frac{2^{l_{i}+l_{f}+1} l_{i} ! l_{f} !}{\left(2 l_{i}+1\right) !\left(2 l_{f}+1\right) !} \frac{\left(l_{i}+l_{f}-4\right) !}{\left[i\left(k_{i}+k_{f}\right)\right]^{l_{i}+l_{f}-3}} \\
& \times k_{i}^{l_{i}+1 / 2} k_{f}^{l_{f}+1 / 2} F_{2}\left(l_{i}+l_{f}-3 ; l_{i}+1, l_{f}+1 ; 2 l_{i}\right. \\
& \left.+2,2 l_{f}+2 ; \frac{2 k_{i}}{k_{i}+k_{f}}, \frac{2 k_{f}}{k_{i}+k_{f}}\right),
\end{aligned}
$$

where $F_{2}$ is the generalized hypergeometric function defined by Appell [26]. The right-hand side of Eq. (10) diverges when $l_{i}+l_{f} \leqslant 3$ due to the singularity of the potential at the origin. When the full potential is taken into account, this unphysical singularity is removed and the $K$-matrix element is unchanged from the $O\left(k_{i}^{l_{i}+1 / 2} k_{f}^{l_{f}+1 / 2}\right)$ behavior that is due to the short-range part of the potential. The right-hand side of Eq. (10) is well behaved when $l_{i}+l_{f}>3$ and provides a modification of the $K$-matrix element when $k_{f}$ is small. For the interesting quasiresonant case $l_{i}=0, l_{f}=4$, and $k_{i} \ll k_{f}$, Eq. (10) reduces to

$$
K_{i f}=\frac{\mu C}{\hbar^{2}} \frac{2 i}{9 ! !}{ }_{2} F_{1}(1,5 ; 10 ; 2) k_{i}^{1 / 2} k_{f}^{7 / 2}\left[1+O\left(k_{i} / k_{f}\right)\right] .
$$

Equation (11) shows that the $K$-matrix element vanishes as $k_{f}^{7 / 2}$ for small $k_{f}$ [note that the hypergeometric function ${ }_{2} F_{1}(1,5 ; 10 ; 2)$ is pure imaginary so the $K$-matrix element is real]. The inelastic scattering cross section therefore vanishes as $k_{f}^{7}$ for small $k_{f}$, and we see that the effect of the longrange interaction is to remove two powers of $k_{f}$ from the threshold behavior produced by the short-range part of the potential. Equation (11) agrees with the result of Nesbet [27] when $\mu=1$ for electron scattering. For ultracold atomdiatom collisions, $\mu=k_{f}^{2} / 2\left(\epsilon_{i}-\epsilon_{f}\right)+O\left(k_{i}^{2}\right)$, where $\epsilon_{i}$ and $\epsilon_{f}$ are the respective diatomic energies in the initial and final channels. Therefore the zero-temperature rate coefficient is given by

$$
\lim _{T \rightarrow 0} R_{v j \rightarrow v^{\prime} j^{\prime}}(T)=\frac{k_{i} \sigma_{v j \rightarrow v^{\prime} j^{\prime}}}{\mu}=O\left(k_{f}^{9}\right) .
$$

The actual momentum dependence of the zerotemperature rate coefficient near threshold will depend on the anisotropy of the potential energy surface. If the anisotropy is weak, then the coefficient $C$ in Eq. (11) will be very small or could even be zero. In order to determine the $k_{f}$-momentum dependence of the $\Delta j=-4 \Delta v$ rate coefficients for $\mathrm{He}-\mathrm{H}_{2}$, we studied the $v=1, j=7$ and $v=3$, $j=7$ threshold regions by smoothly varying the mass of the helium atom. This is equivalent to varying $k_{f}^{2}$ in the limit that $k_{i} \rightarrow 0$. The results are given in Figs. 8 and 9. For the initial $v=1, j=7$ state we find that the zero-temperature rate coefficients behave like $k_{f}^{9}$ for small $k_{f}$. The good agreement between this fit and the exact curve when $k_{f}$ is less than 2 $\AA^{-1}$ indicates that the long-range part of the potential has a strong influence on the threshold behavior. For the initial $v$ $=3, j=7$ state, we find that the zero-temperature rate coefficients also behave like $k_{f}^{9}$ for small $k_{f}$, but that it is necessary to include an additional $O\left(k_{f}^{11}\right)$ term in order to obtain good agreement with the exact curve. Therefore we conclude that the long-range part of the potential has a weak influence on the threshold behavior for this case.

Figures 8 and 9 also show that a sharp spike appears in the rate coefficients when the reduced mass $\mu$ sweeps below $1.2 \mathrm{amu}$. This spike occurs when the most weakly bound state of the van der Waals complex approaches zero energy. These so-called zero-energy resonances have a strong influence on both the elastic and inelastic scattering cross sections. Using the asymptotic analysis developed previously [13] we may show that the function

$$
f_{v j}(\mu)=\frac{\beta_{v j}}{\alpha_{v j}^{2}}
$$




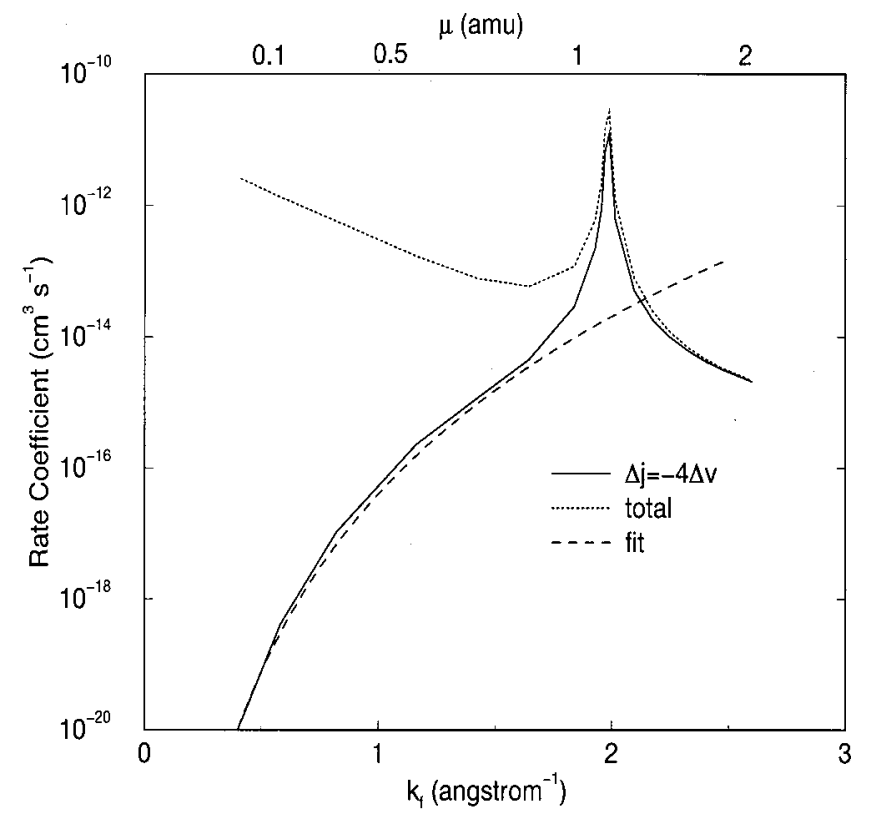

FIG. 8. Quantum-mechanical calculation of the zerotemperature rate coefficient for initial $v=1, j=7$ making a $\Delta j=$ $-4 \Delta v$ transition (solid curve). The total quenching rate coefficient (dotted curve) decays exponentially with increasing $k_{f}$ on the left side of the zero-energy resonance. The $\Delta j=-4 \Delta v$ transition is the dominant transition when $k_{f}$ is greater than the position of the resonance and the $\Delta j=-2, \Delta v=0$ transition is the dominant transition when $k_{f}$ is smaller than the position of the resonance. Also shown is a fit (dashed curve) which is proportional to $k_{f}^{9}$ in agreement with Eq. (11).

is analytic in the vicinity of the zero-energy resonance. This was confirmed by numerical tests which showed that $\alpha_{v j}$ $\propto\left(\mu-\mu_{0}\right)^{-1}$ and $\beta_{v j} \propto\left(\mu-\mu_{0}\right)^{-2}$ for $\mu$ near $\mu_{0}$ $=1.16 \mathrm{amu}$.

An interesting observation may be made from Figs. 8 and 9 regarding the nearly linear behavior (on the logarithmic scale) of the total quenching rate coefficient on the left side of the zero-energy resonance. On the left side of the resonance, the total quenching rate coefficient is dominated by the $\Delta j=-2, \Delta v=0$ transition. The energy gap for this transition produces a $k_{f}$ value that is much larger than those shown on the horizontal axis of the figure (the $k_{f}$ values shown on the figure are for the $\Delta j=-4 \Delta v$ energy gap). Figures 8 and 9 show that when the quasiresonant transition is on the threshold of closing, the dominant quenching rate coefficient tends to follow an exponential dependence with the momentum gap. Because the total quenching rate coefficient at zero temperature is inversely related to the predissociation lifetime of the most weakly bound state [see Eq. (2)], this analysis would predict an exponential dependence on the momentum gap for predissociation. This is in agreement with the well-known "exponential momentum gap law" which may be derived using Eq. (1) and is a consequence of the Franck-Condon overlap between the vibrational wave function of the bound complex and the continuum wave function of the fragments $[18,19]$. The exponential gap law assumes that the transition is the result of a direct process. On the right side of the zero-energy resonance, however, it is

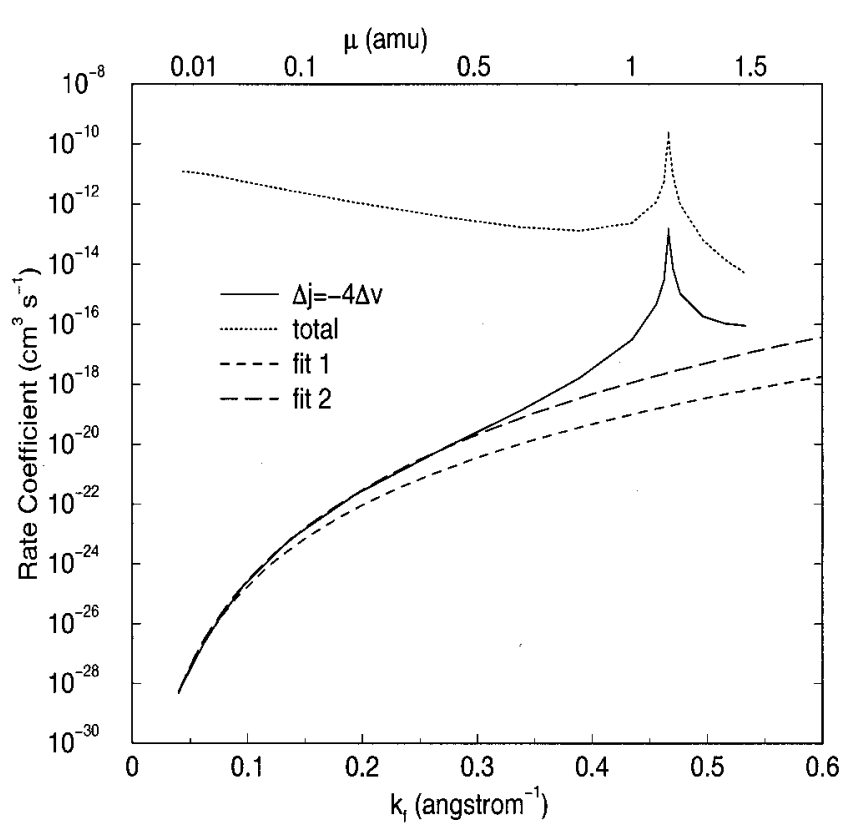

FIG. 9. Quantum-mechanical calculation of the zerotemperature rate coefficient for initial $v=3, j=7$ making a $\Delta j$ $=-4 \Delta v$ transition (solid curve). The total quenching rate coefficient (dotted curve) decays exponentially with increasing $k_{f}$ on the left side of the zero-energy resonance where $\Delta j=-2, \Delta v=0$ transitions are dominant. Also shown are fit 1 (dashed curve) and fit 2 (long dashed curve) which are proportional to $k_{f}^{9}$. An additional term proportional to $k_{f}^{11}$ has been included in fit 2 .

the quasiresonant $\Delta j=-4 \Delta v$ transition that is dominant. The predissociation lifetimes may be obtained from these rate coefficients using Eq. (2) as above. In this case, however, the predissociation lifetimes do not exhibit exponential dependence on the momentum gap because of the importance of the indirect contributions.

\section{CONCLUSION}

It has been known for some time [17-21] that a balance exists between momentum gap and near-resonant effects in the vibrational predissociation of weakly bound complexes. It has also been shown that high-order indirect potential couplings must be influencing the predissociation [21]. In the present work, we considered the special case where the van der Waals molecule is comprised of a vibrating diatom that is also in a state of high rotation. For this case, we showed that the process of predissociation for the most weakly bound state of a van der Waals complex is controlled by the same quasiresonant transfer of energy that is found in atom-diatom collisions whenever the quasiresonant channel is energetically open. The conservation of classical action provides an understanding of the mechanism underlying the high-order potential coupling. This is consistent with the view [28] that underlying the quantum-mechanical atom-molecule scattering is "a coarser graining rooted in the classical mechanics of the collision." The lifetimes are also strongly influenced by the proximity of closed channel thresholds. We studied the analytic structure of the threshold behavior and made 
predictions that may be experimentally tested with the use of trapped molecules. Since the effective range theory that was used to relate the collisional rate coefficients to the predissociation lifetimes applies only to weakly bound states, it will be interesting to see whether quasiresonant behavior is also found in the more deeply bound states of the van der Waals complex. We leave this as a subject for future investigation.

\section{ACKNOWLEDGMENTS}

The research of R.C.F. was supported by National Science Foundation Grant No. PHY-0070920. The research of A.D. was supported by the Chemical Sciences, Geosciences and Biosciences Division of the Office of Basic Energy Sciences, Office of Science, U.S. Department of Energy.
[1] J. D. Weinstein, R. deCarvalho, T. Guillet, B. Friedrich, and J. M. Doyle, Nature (London) 395, 148 (1998); J. Doyle and B. Friedrich, Chem. Br. 35, 31 (1999).

[2] J. T. Bahns, P. L. Gould, and W. C. Stwalley, Adv. At., Mol., Opt. Phys. 42, 171 (2000).

[3] A. Fioretti, D. Comparat, A. Crubellier, O. Dulieu, F. MasnouSeeuws, and P. Pillet, Phys. Rev. Lett. 80, 4402 (1998).

[4] A. N. Nikolov, J. R. Ensher, E. E. Eyler, H. Wang, W. C. Stwalley, and P. L. Gould, Phys. Rev. Lett. 84, 246 (2000); A. N. Nikolov, E. E. Eyler, X. Wang, H. Wang, J. Li, W. C. Stwalley, and P. L. Gould, ibid. 82, 703 (1999).

[5] T. Takekoshi, B. M. Patterson, and R. J. Knize, Phys. Rev. A 59, R5 (1999); Phys. Rev. Lett. 81, 5109 (1998).

[6] H. L. Bethlem, G. Berden, and G. Meijer, Phys. Rev. Lett. 83, 1558 (1999); H. L. Bethlem, G. Berden, A. A. van Roij, F. M. H. Crompvoets, and G. Meijer, ibid. 84, 5744 (1999).

[7] J. A. Maddi, T. P. Dinneen, and H. Gould, Phys. Rev. A 60, 3882 (1999).

[8] M. Gupta and D. Herschbach, J. Phys. Chem. A 103, 10670 (1999).

[9] J. M. Gerton, D. V. Strekalov, I. D. Prodan, and R. G. Hulet, Bull. Am. Phys. Soc. 45, 13 (2000).

[10] C. J. Williams and P. S. Julienne, Science 287, 986 (2000).

[11] R. Wynar, R. S. Freeland, D. J. Han, C. Ryu, and D. Heinzen, Science 287, 1016 (2000).

[12] N. Balakrishnan, R. C. Forrey, and A. Dalgarno, Phys. Rev. Lett. 80, 3224 (1998); Chem. Phys. Lett. 280, 1 (1997); N. Balakrishnan, A. Dalgarno, and R. C. Forrey, J. Chem. Phys. 113, 621 (2000).

[13] R. C. Forrey, V. Kharchenko, N. Balakrishnan, and A. Dalgarno, Phys. Rev. A 59, 1 (1999); R. C. Forrey, N. Balakrishnan, V. Kharchenko, and A. Dalgarno, ibid. 58, R2645 (1998).
[14] R. C. Forrey, N. Balakrishnan, A. Dalgarno, M. Haggerty, and E. J. Heller, Phys. Rev. Lett. 82, 2657 (1999).

[15] J. Weiner, V. S. Bagnato, S. Zilio, and P. S. Julienne, Rev. Mod. Phys. 71, 1 (1999).

[16] J. L. Bohn, Phys. Rev. A 6203, 2701 (2000).

[17] M. S. Child and C. J. Ashton, Faraday Discuss. Chem. Soc. 62, 307 (1977).

[18] J. A. Beswick and J. Jortner, J. Chem. Phys. 68, 2277 (1978); Adv. Chem. Phys. 47, 363 (1981).

[19] G. E. Ewing, Chem. Phys. 29, 253 (1978); J. Chem. Phys. 71, 3143 (1979); 72, 2096 (1980).

[20] R. J. LeRoy, G. C. Corey, and J. M. Hutson, Faraday Discuss. Chem. Soc. 73, 339 (1982).

[21] J. M. Hutson, C. J. Ashton, and R. J. LeRoy, J. Phys. Chem. 87, 2713 (1983).

[22] T. Takayanagi and Y. Kurosaki, Chem. Phys. Lett. 286, 35 (1998); T. Takayanagi and Y. Kurosaki, J. Chem. Phys. 109, 8929 (1998).

[23] J. M. Hutson and S. Green, MOLSCAT computer code, version 14 (1994), distributed by Collaborative Computational Project No. 6 of the Engineering and Physical Sciences Research Council (UK).

[24] P. Muchnick and A. Russek, J. Chem. Phys. 100, 4336 (1994).

[25] B. Stewart, P. D. Magill, T. P. Scott, J. Derouard, and D. E. Pritchard, Phys. Rev. Lett. 60, 282 (1988); P. D. Magill, B. Stewart, N. Smith, and D. E. Pritchard, ibid. 60, 1943 (1988).

[26] A. Erdelyi, Higher Transcendental Functions (McGraw-Hill, New York, 1953), Vol. 1.

[27] R. K. Nesbet, Variational Methods in Electron-Atom Scattering Theory (Plenum Press, New York, 1980).

[28] A. J. McCaffery and R. J. Wilson, Phys. Rev. Lett. 77, 48 (1996). 